\title{
Guiding Lights in Genome Editing for Inherited Retinal Disorders: Implications for Gene and Cell Therapy
}

\author{
Carla Sanjurjo-Soriano ${ }^{1,2}$ and Vasiliki Kalatzis (D) $^{1,2}$ \\ ${ }^{1}$ Inserm U1051, Institute for Neurosciences of Montpellier, Montpellier, France \\ ${ }^{2}$ University of Montpellier, Montpellier, France \\ Correspondence should be addressed to Vasiliki Kalatzis; vasiliki.kalatzis@inserm.fr
}

Received 26 January 2018; Accepted 18 April 2018; Published 8 May 2018

Academic Editor: Melissa R. Andrews

Copyright (c) 2018 Carla Sanjurjo-Soriano and Vasiliki Kalatzis. This is an open access article distributed under the Creative Commons Attribution License, which permits unrestricted use, distribution, and reproduction in any medium, provided the original work is properly cited.

\begin{abstract}
Inherited retinal dystrophies (IRDs) are a leading cause of visual impairment in the developing world. These conditions present an irreversible dysfunction or loss of neural retinal cells, which significantly impacts quality of life. Due to the anatomical accessibility and immunoprivileged status of the eye, ophthalmological research has been at the forefront of innovative and advanced gene- and cell-based therapies, both of which represent great potential as therapeutic treatments for IRD patients. However, due to a genetic and clinical heterogeneity, certain IRDs are not candidates for these approaches. New advances in the field of genome editing using Clustered Regularly Interspaced Short Palindromic Repeats (CRISPR) and CRISPR-associated protein (Cas) have provided an accurate and efficient way to edit the human genome and represent an appealing alternative for treating IRDs. We provide a brief update on current gene augmentation therapies for retinal dystrophies. Furthermore, we discuss recent advances in the field of genome editing and stem cell technologies, which together enable precise and personalized therapies for patients. Lastly, we highlight current technological limitations and barriers that need to be overcome before this technology can become a viable treatment option for patients.
\end{abstract}

\section{Introduction}

The eye, and more specifically the retina, as an extension of the central nervous system (CNS), provides a powerful and unique "window" to study neuronal diseases. The retina shares anatomical and developmental characteristics with the brain [1]. For example, it is relatively immunoprivileged and has specialized immune responses similar to the ones found in the brain and spinal cord [2,3]. In addition, it is surrounded by the inner blood-retinal barrier (BRB), which is composed of the same nonfenestrated endothelial cells as those found in the blood-brain barrier (BBB) [4]. Due to the accessibility of the eye by modern techniques of vitreoretinal surgery, it is not surprising that major research and understanding in the context of the CNS has emerged from studies of the retina and the optic nerve [5-11]. Furthermore, the significant compartmentalization of the eye, and specifically the retina, has allowed it to become a prototype for the development of innovative therapies and has brought ocular diseases to the forefront of clinical translation for gene- and cell-based therapies. Here, we will specifically review current progress in these therapeutic strategies for diseases of the posterior retina (namely the neuronal photoreceptor cells). Optic neuropathies affecting the anterior retina (retinal ganglion cells (RGCs)) and optic nerve are beyond the scope of this review.

\section{The Retina}

The retina is an embryonic extension of the prosencephalon [12]. It lines the back of the eye and consists of multiple cell layers that are responsible for the detection and processing of visual information. The retina has a highly structured architecture that can be divided into a posterior pigmented monolayer and an anterior multilayered neuroretina. The posterior layer, the retinal pigment epithelium (RPE), plays an important role in protection (excess light absorption, phagocytosis, water and ion transport) and support (growth 


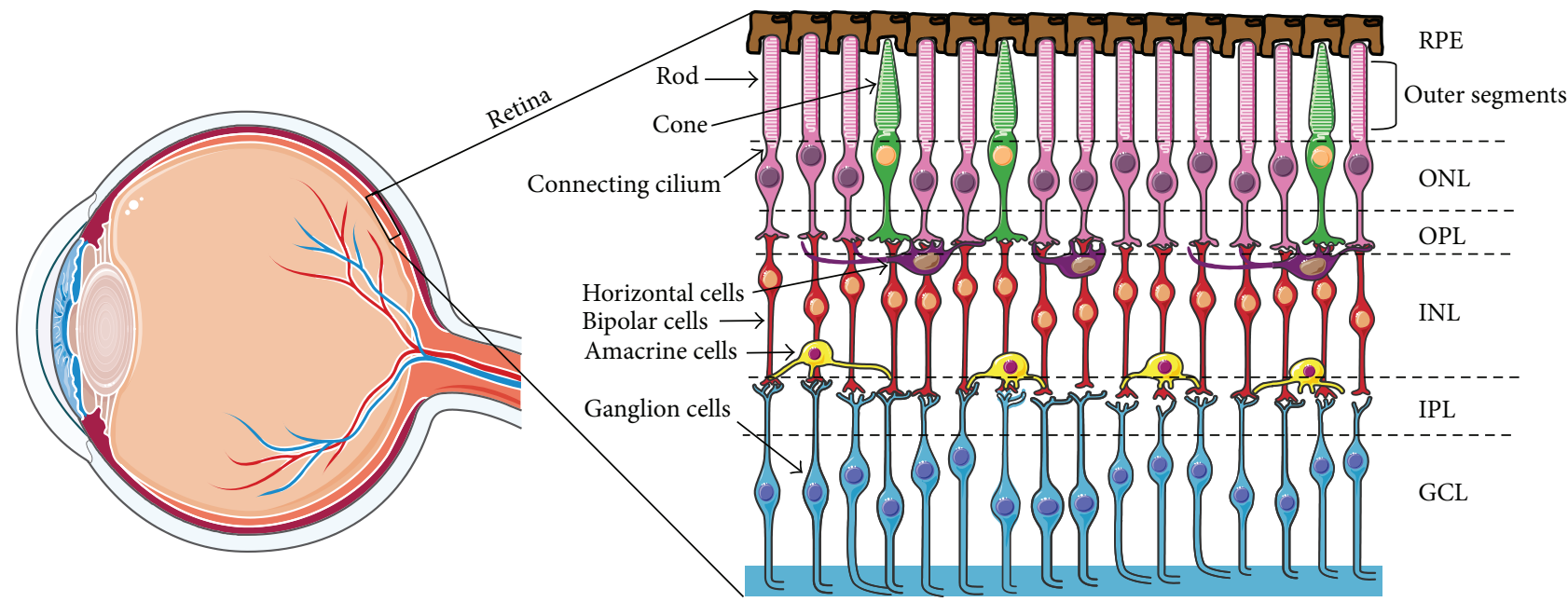

FIGURE 1: Schematic representation of the retina and the retinal cell layers. The retina is a layered structure lining the back of the eye consisting of a pigmented layer, the RPE, and a multilayered neuroretina. The RPE is in close contact with the outer segments of the photosensitive rod and cone cells of the neuroretina. The connecting cilium connects the photoreceptor outer segments with the cell bodies, which constitute a layer known as the outer nuclear layer (ONL). The axons of the photoreceptors synapse with the neuronal (bipolar, amacrine, and horizontal) cells of the inner nuclear layer (INL) via the outer plexiform layer (OPL). The axons of the INL cells in turn synapse with the ganglion cell layer (GCL) via the inner plexiform layer (IPL). The axons of the ganglion cells converge to form the optic nerve.

factor section, nutrient transport) of the photoreceptor layer $[13,14]$. The neuroretina is highly stratified, and it is composed of three layers of specialized neurons that are interconnected by two synaptic layers (Figure 1). The first layer comprises the photosensitive rod and cone photoreceptor cells with their characteristic outer segments, within which the phototransduction process that follows light interaction takes place. Light intensity dictates which photoreceptor cells are used. In bright light, it is the centrally prevalent cones, and in low light, it is the peripherally prevalent rods. The photoreceptors then synapse with interneurons within the second layer, which transmit the electrical signal arriving from the photoreceptors to the RGCs in the third layer [15]. The axons of the RGCs form a nerve fibre layer, which becomes the optic nerve, and hence, the signal is transmitted from the eye to the brain for image interpretation. The inability to convert the light signal and transmit the electrical signal to the brain is the primary cause of visual impairment in the developing world. A large proportion of cases is due to dysfunction and/or loss of photoreceptors caused by a series of risk factors including age, diabetes, and genetics [16]. The latter gives rise to a specific subset of conditions referred to as inherited retinal dystrophies.

\section{Inherited Retinal Dystrophies}

Inherited retinal dystrophies (IRDs) are a genetically and clinically heterogeneous group of neurodegenerative disorders that lead to progressive visual impairment $[16,17]$. They affect approximately 1 in 2000 individuals worldwide [18]. IRDs have been associated with mutations in more than 250 genes (see http://www.sph.uth.tmc.edu/Retnet), affecting the development, function and/or survival of the photoreceptors, and RPE [19], and with autosomal dominant, recessive, or X-linked transmission [16]. Furthermore, complex, multifactorial, and heterogeneous diseases such as age-related macular degeneration (AMD) are also considered retinal dystrophies.

IRDs can be divided into nonsyndromic forms, characterized by an isolated retinal phenotype, or syndromic forms, in which another organ in addition to the eye is affected. Nonsyndromic IRDs can be further broken down into subgroups based on the disease progression and the region of the retina that is affected. Firstly, progressive conditions affecting exclusively the central retina (macula), leading to central vision loss, are known as macular dystrophies. The most common example is Stargardt disease with a prevalence of $1 / 10000$, which is due to mutations in the gene $A B C A 4$ [20]. Secondly, progressive conditions affecting the retina more widely can be classified depending on the type of photoreceptor that degenerates initially. Rod-cone dystrophies, where the rods are first affected, are characterized initially by night blindness and subsequently by peripheral vision loss; the most prevalent example (1/4000) is retinitis pigmentosa (RP), caused by mutations in over 80 genes [21]. By contrast, in cone-rod dystrophies, the cones are first affected, leading to decreased sharpness of visual acuity and blind spots in the center of the visual field; $A B C A 4$ mutations also account for the majority of these cases [22].

When both the macula and the peripheral retina are affected and there is a rapid retinal degeneration from birth, the condition is known as Leber congenital amaurosis (LCA; prevalence of 1/50000), of which 18 types are recognized. In addition, if the retinal changes are associated with a degeneration of the choroid, a highly vascular, pigmented tissue underlying the retina, these diseases are referred to as chorioretinopathies. Choroideremia (CHM) is the most common example (prevalence of 1/50000) in this group. The most 
common form of syndromic IRD is the heterogeneous Usher syndrome group (prevalence of $1 / 20000$ ), which is characterized by RP and hearing loss [23]. Usher syndrome is further subdivided into three subtypes depending on the severity and progression of the hearing loss and the age of onset of the RP. Usher syndrome type 1 (USH1) is the most severe; Usher syndrome type 2 (USH2) is the most common presenting moderate to severe symptoms. Lastly, Usher syndrome type 3 (USH3) presents a moderate phenotype and variable progression and onset of the disease.

The monogenic nature of IRDs coupled to the accessibility and immunoprivileged nature of the human eye has led to the advancement of pioneer gene therapies that hold promise for the development of future treatments. Most predominantly, IRDs have been targets for gene augmentation therapy [24]. More recently, gene correction of the causative gene, either by inactivation of the autosomal dominant allele or by correction of the recessive or X-linked alleles, has been explored as a possible treatment strategy. Currently, there is no standardized therapeutic option in the clinic for IRDs, due to the challenges of a diverse genetic landscape, fluctuating disease prevalence, variable age of onset and clinical course, and the specificity of the therapeutic products.

\section{Gene Augmentation Therapy for IRDs}

Gene augmentation therapy provides a normal copy of a mutated gene into native cells and hence is applicable for the treatment of haploinsufficiency or loss-of-function mutations. Most commonly, but not exclusively, the genes are vehicled by viral vectors, a pertinent example being adenoassociated viral (AAV) vectors [25]. AAV vectors present specific characteristics such as low immunogenicity and toxicity, lack of pathogenicity, long-term transgene expression, and relative ease in manipulating genetic elements, making them the safest and most effective viral vector platform for gene delivery into the retina to date [26]. Delivery can be achieved by subretinal injection, where the vector is administered into the subretinal space between the photoreceptors and RPE, which can result in the transduction of both cell types depending on the serotype used [27]. Other methods, such as intravitreal delivery, are less invasive and thus result in fewer complications postsurgery, but the delivery of the therapeutic genes, particularly to the posterior retina, is less effective [28].

A major milestone in gene augmentation therapy for IRDs was achieved in 2001 using a canine model for LCA2 due to mutations in the gene RPE65 $\left(R^{2} E 65^{-1-}\right)$. AAV2/2mediated delivery of RPE65 led to the long-term restoration of vision in treated dogs [29]. Following this study, multiple phase $1 / 2$ clinical gene therapy trials assessed the effects of subretinal administration of AAV-RPE65 and demonstrated improved vision in some patients with no adverse effects of the vector [30-34]. A phase 3 clinical trial for LCA2, in which the therapeutic vector was administrated in both eyes, was subsequently launched. The vision of the treated group significantly increased compared to the control group, and this became the first ocular clinical trial in which both eyes were treated successfully [35]. As a consequence, the corresponding vector has been recently commercialized as a drug under the name of voretigene neparvovec (Luxturna).

Hot on the heels of the AAV-RPE65 trial, phase 1/2 clinical trials for the X-linked chorioretinopathy choroideremia were initiated $[36,37]$ following preclinical studies in $\mathrm{Chm}^{\text {null/WT }}$ mice [38]. These trials are also using an AAV2/ 2 vector, administered subretinally, to vehicle the causative CHM gene into both photoreceptors and the RPE. However, preclinical studies have shown that other AAV serotypes such as AAV2/5 [39] and AAV2/8 [40] are also effective for choroideremia. Lastly, a phase 1 clinical trial to treat RP was performed using an AAV2/2 vector to vehicle the causative gene MERTK [41] confirming the safety profile of this vector serotype. A variety of other clinical trials have been initiated worldwide for other IRD genes using alternative AAV serotypes, but the results are still forthcoming.

Despite its numerous advantages, AAV vectors are limited by their cloning capacity $(<4.7 \mathrm{~kb})[42-44]$. To overcome this limitation, efforts have turned to the use of equine infectious anemia virus- (EIAV-) based lentiviral vectors, which although integrative are nonpathogenic to humans. An EIAV vector was first tested in the case of the $A B C A 4$ gene, which has a $6.8 \mathrm{~kb}$ coding sequence. Preclinical studies in the mouse $A b c a 4^{-1-}$ model showed a reduction in toxic A2E accumulation in the RPE of treated mice as compared to controls [45]. Following biodistribution and safety studies of the corresponding EIAV ABCA4-carrying vector [46], a clinical trial was begun in 2011, but the results are still pending. Similarly, an EIAV vector, carrying the MYO7A gene $(6.5 \mathrm{~kb})$, was tested for its efficiency in the treatment of RP associated with Usher syndrome 1B [47]. Proof-of-concept studies in the mouse $M y o 7 A^{-1-}$ model suggested that the vector was able to prevent light-induced retinal degeneration [48]; however, the results of the clinical trial begun in 2012 are also pending. The outcome of these two EIAV clinical trials is essential to assess the suitability of lentiviral-based vectors for therapy of IRDs due to large causative genes.

Since the landmark canine LCA2 study by Acland et al., the progress in precision medicine research has continued to develop. However, several challenges remain to be overcome. Despite variations in visual improvements among treated patients in the LCA2 trials, long-term follow-up studies showed that the retinal structure continued to degenerate $[49,50]$. This could be attributed to the advanced disease course at the time of treatment at which point the degeneration process could no longer be halted [51-53]. Advanced stages of retinal degeneration are incompatible with gene augmentation therapy, which, to be successful, requires that the nonfunctional target cells are still alive. Such patients might benefit better from cell-based transplantation therapy, which has the potential to restore visual function as detailed later.

An alternative explanation for the continual degeneration posttreatment could be inefficient vector transduction $[52,54]$. Achieving correct levels of gene expression is essential for a robust and significant rescue of the phenotype $[50,55,56]$. This may be improved by the use of alternative $[39,57,58]$ or modified [59] AAV serotypes, which have been shown to have a higher transduction efficiency than 


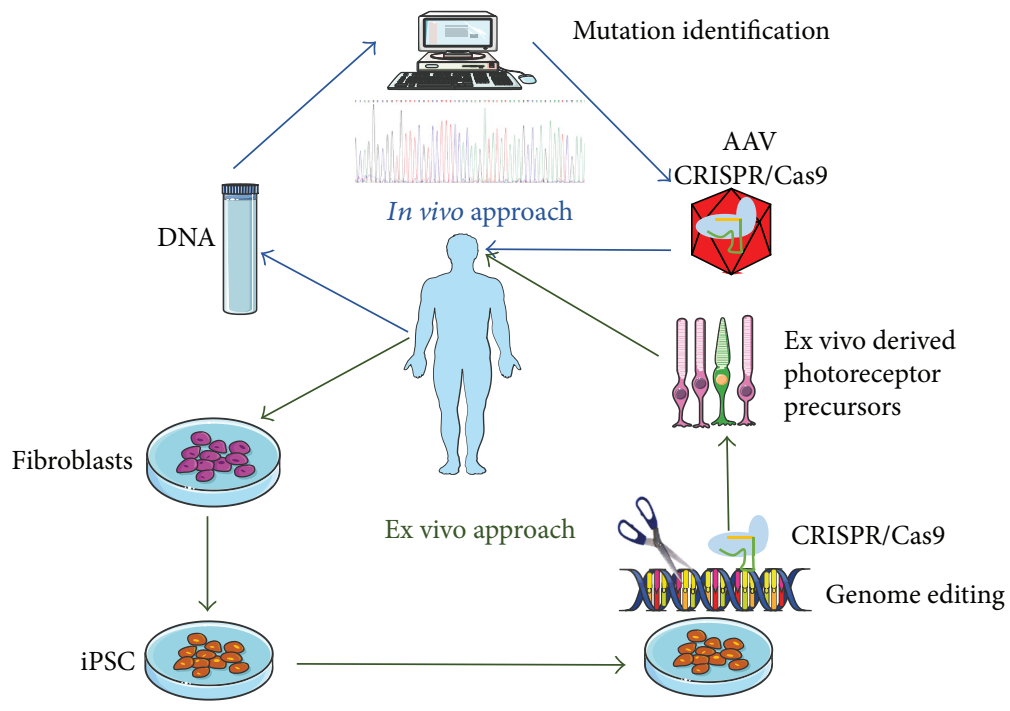

FIGURE 2: Therapeutic approaches for treating retinal dystrophies. For an in vivo approach (indicated in blue), the patient's DNA is isolated, and genetic screening is carried out to identify the pathogenic mutation causing the retinal phenotype. Delivery of the CRISPR/Cas9 components to correct the pathogenic mutation in vivo is achieved via AAV vectors administrated directly to the retina of the patients. For an ex vivo approach (in green), patient's fibroblasts with a known mutation in an IRD gene are isolated and reprogrammed to patient-specific iPSC. Genome editing of iPSCs is carried out using the CRISPR/Cas9 system. The corrected iPSCs are further differentiated into retinal cells, which can then be reimplanted into the patient's retina.

AAV2/2 in multiple species, or optimized promoter and/or codon-optimized cDNA sequences, which can stabilize transcript expression and hence increase protein levels [60,61]. Finally, gene augmentation strategies are not convenient approaches for treating dominant or dominant-negative mutations, in which the mutated allele causing the disease needs to first be inactivated so that it does not interfere with the wild-type copy [62]. This is generally most easily accomplished by dual (wild-type and mutant) allele silencing prior to gene augmentation [63-66]. Therefore, despite the limited benefit demonstrated in clinical trials using AAV-mediated retinal gene augmentation therapy for the treatment of recessive mutations, other approaches for treating IRDs are being investigated with promising results.

\section{Genome Editing for the Treatment of IRDs}

Providing a wild-type copy of the mutated allele to restore a phenotype does not directly impact the pathogenic host gene. In contrast, a genome-editing approach has the potential of correcting the mutation directly in the patient's DNA. This approach could thus fill the void left by gene augmentation therapy in the case of large causative genes or dominant mutations. There are potentially two different approaches in the case of genome editing: an in vivo approach whereby the disease-causing mutations are corrected directly in the retina and an ex vivo approach in which the mutation is corrected in the patient's cells in view of future cell transplantation (Figure 2). The advances and current progress for both strategies will be summarized here. In addition to correcting pathogenic mutations, genome editing has also been used in a variety of preclinical models to further understand disease pathogenesis and to determine feasible treatment options.
Genome editing has advanced at an exceptionally rapid rate, creating huge impacts on biotechnology and biomedicine. The genome-editing era was initially triggered by the use of engineered meganucleases and zinc finger nucleases (ZFN) to specifically target a genomic sequence. Later, the development of transcription activator-like effector nucleases (TALEN) and more recently, the Clustered Regularly Interspaced Short Palindromic Repeats (CRISPR) and CRISPRassociated genes (Cas) system have led to a scientific genome-editing revolution.

5.1. ZFNs and TALENs. Efficient genome editing, regardless of which tool is used, is based upon the introduction of a double-strand break (DSB) at a precise point in the genome, which rapidly stimulates one of the two DNA repair pathways of the cell $[67,68]$. The nonhomologous end-joining (NHEJ) pathway is the default method of repair, introducing insertions and deletions (INDELs) that normally will result in a nonfunctional genetic product [69]. Alternatively, homology-directed repair (HDR) uses the sister chromatids from a homologous chromosome as a template, or, in the case of directed genome editing, a donor template containing the desired sequence [70] (Figure 3). HDR occurs much less frequently than NHEJ, since homologous recombination naturally occurs in the late $S$ and $G 2$ phases of cellular division [71].

To induce a DSB, ZFNs and TALENs need to be guided to the target sequence by a protein DNA-binding domain. They therefore rely on the engineering of new proteins for each target, which has made genome editing difficult, laborious, and challenging [72]. Zinc finger proteins are a class of transcription factors that bind DNA through Cys2-His2 zinc finger domains [73]. ZFNs consist of a modifiable zinc finger domain designed to bind and target specific sequences in the 


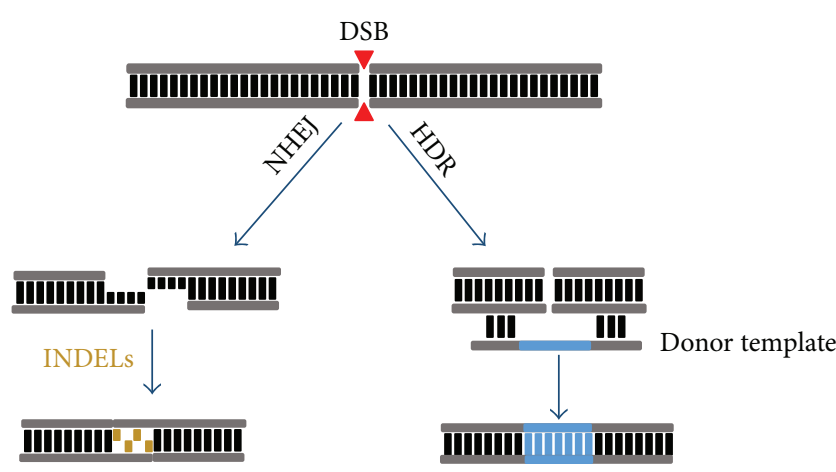

FIGURE 3: Schematic representation of a double-stranded break (DSB; red arrowheads), which can be repaired through nonhomologous end-joining (NHEJ) or homology-directed repair (HDR) pathways. The introduction of a double-strand break in the DNA will typically undergo the error-prone NHEJ repair pathway, which results in insertions and deletions (INDELs) of variable length that will lead to premature stop codon formation. $\mathrm{HDR}$, an error-free repair pathway, occurs using a wild-type donor template with homology to the target site, which serves as a template for precise gene correction of the host's DNA.

genome and a cleavage domain consisting of the FokI nuclease $[74,75]$. The cleavage of the DNA at the desired site is triggered by the dimerization of FokI; thus, two sets of ZFN on either side of the cleavage site are needed for the introduction of the DSB [76]. Similarly, TALENs are engineered by fusing a TAL effector DNA-binding domain with a FokI nuclease cleavage domain $[77,78]$. TAL proteins are made of tandem repeats binding to individual nucleotides, which is different to ZFNs in which a zinc finger domain can bind to three different nucleotides (Figure 4). TALENs emerged as an alternative to ZFNs, as they represented a quicker turnaround from design to implementation and a more affordable option. Nonetheless, TALENs are relatively large proteins and contain repetitive DNA sequences resulting in TALEN inactivation [79], making genome editing still very challenging for researchers. In addition, similar to ZFNs, engineering of novel proteins for each DNA target is required.

Despite the challenges, ZFNs have been used as a proof-of-concept treatment for retinal disease. Human embryonic retinoblast cells expressing the Pro23His mutation in the Rhodopsin (RHO) gene were targeted with ZFNs. An increase in homologous recombination events occurred when the ZFNs were transfected with a homologous donor template compared to delivery of the ZFNs alone [80]. Similarly, researchers achieved site-specific gene correction in HEK293 cells stably expressing a missense mutation in Ush1c, causing Usher syndrome 1C. The authors reported correction of the pathogenic mutation by homologous recombination triggered by ZFNs and a donor plasmid template, when both were transfected to the cells [81]. These studies were the first to demonstrate the feasibility of gene targeting for retinal dystrophies using ZFNs. The major limitation for the applicability of ZFN relies on the design of the zinc fingers to bind every combination of three base pairs present in the genome, which has not yet been achieved.
Thus, many sites cannot be targeted using these engineered nucleases [77]. TALEN engineering has also been applied to the retina for the correction of a mutation in the $C r b 1^{r d s}$ mouse, a model for LCA8. The mouse oocytes were treated with mRNA-encoding TALENs targeting the $\mathrm{Crbl}^{\text {rds }}$ allele together with a single-stranded oligonucleotide (ssODN) to correct the pathogenic allele. HDR triggered by TALEN and ssODN repair template was observed in $27 \%$ of the treated mice embryos, which presented an improvement of the ocular defects [82].

5.2. CRISPR/Cas Systems. The CRISPR/Cas system represents a novel and efficient method for genome editing compared to ZFNs and TALENs. CRISPR were first noticed in the bacterial genome in 1987 and described as an "unusual structure" in the $3^{\prime}$ region of the iap gene, containing 29base pair repeats interspaced by 32 nonrepetitive nucleotides [83]. Later, similar repeats were found in numerous bacteria and archaea [84-86]. It was in 2000 when the acronym CRISPR was given to unify these repeats observed in the bacterial genome $[87,88]$. In addition, researchers discovered several clusters of protein-coding genes adjacent to these repeats, and they were subsequently called CRISPRassociated genes or Cas genes [87]. Evidence emerged that CRISPR loci might be involved in bacterial immunity, but it was not until 2007 when it was demonstrated that the CRISPR/Cas system provides resistance against specific phages in the bacterial strain Streptococcus thermophiles [89].

CRISPR as a genome editing-system was first described in 2012 [90]. Jinek and colleagues found that the CRISPR/ Cas system of Streptomyces pyogenes (spCas9) was capable of inducing a DSB when two RNA molecules were present, a CRISPR RNA (crRNA) and a trans-activating RNA (tracrRNA). In addition, the authors showed that the fusion of the crRNA and tracrRNA produces a single-guide RNA (gRNA), which is equally effective in binding to target DNA. At the $5^{\prime}$ end of this fused gRNA, 20 nucleotides can be customized to target specific sequences, becoming the first requirement for site-specific genome editing using CRISPR technology [90]. A second requirement for precise genome editing is found at the $3^{\prime}$ end directly downstream of the cleavage site, where the protospacer adjacent motif (PAM), a three-nucleotide sequence (NGG in the case of SpCas9), is an absolute requirement for Cas9 recognition (Figure 5) [91]. The combination of both, the gRNA and the PAM sequence, allows target-specific cleavage of the DNA triggered by the Cas9 endonuclease [92]. Not long after these developments, the system was used to provide efficient gene repair in cells and in numerous organisms [70, 93-97].

\subsection{Developments and Advances in CRISPR/Cas Technology.} Since the emergence of the CRISPR/Cas9 technology, researchers have focused on the development of more efficient Cas9-like nucleases, presenting similar on-target activity but reduced off-target activity. A limiting factor for the reduction of off-targets triggered by the Cas endonuclease is the cellular levels of Cas9 protein in the cells. It has been shown that high levels increase the likelihood of off-target cleavage, most likely due to the increase in mismatch 


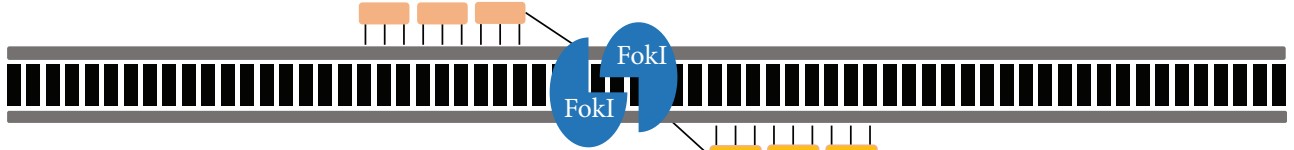

(a)

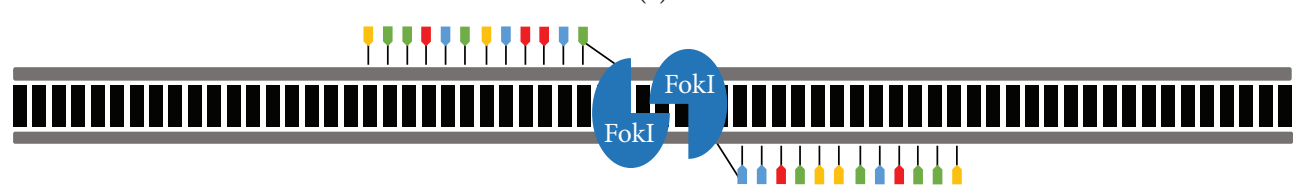

(b)

FIgURE 4: Schematic representation of the structure of a zinc finger nuclease (ZFN) and transcription activator-like effector nucleases (TALENs). (a) Cartoon of a ZFN dimer bound to DNA. ZFNs consist of two functional domains. A DNA-binding domain composed of three zinc finger modules, each one recognizing a unique triplet $(3 \mathrm{bp})$ in the DNA. The DNA-cleaving domain composed of the FokI nuclease is attached to the zinc finger modules and induces the DSB in the DNA. (b) Cartoon of a TALEN dimer bound to DNA. TALENS bind DNA using the TAL effector recognizing individual nucleotides forming the DNA-binding domain. In addition, a DNAcleaving domain comprised of the FokI nuclease is also present and will induce the DSB at the precise location in the DNA.

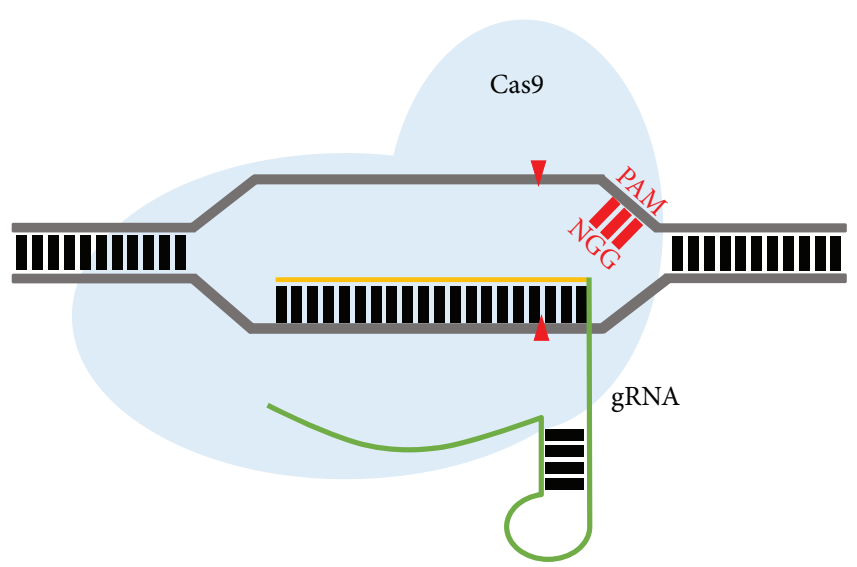

FIGURE 5: Schematic representation of the CRISPR/Cas9 system. The Streptococcus pyogenes Cas9 nuclease, with a "NGG" protospacer adjacent motif (PAM) sequence, has been targeted to a 20 -nucleotide guide sequence in a specific region in the genome (yellow). The gRNA is complementary to the non-PAM strand. The green line represents the gRNA scaffold, which complexes with the Cas9 nuclease (light blue) and directs it to the desired site to induce a DSB (red arrowheads) in the DNA. Cas9 mediates the DSB 3 bp upstream of the PAM sequence.

tolerance between the gRNA and the DNA [98-100]. Successful efforts to overcome this limitation have been the delivery of Cas 9 as a purified protein instead of using expression plasmids with strong promoters [101-105]. Alternatively, limiting the duration of Cas9 expression in the targeted cells has also been investigated. This approach has been successfully achieved in the retinal landscape as described below, presenting a huge advantage for future in vivo genome editing for eye diseases [106]. The use of two gRNA flanking the target region can also increase the on-target activity while reducing off-target events [92]. This strategy known as nickase Cas9 can be achieved by inactivation of one of the two nuclease domains of the Cas9, resulting in the cleavage of only one DNA strand. This strategy reduces the off-target DNA cleavage rate by 50 - to 1500 -fold as compared to a DSB performed at the same sequence [92]. Recent advances have come with the development of Cas9 mutants, which decrease nonspecific DNA interactions. Two parallel studies developed rationally altered spCas9 mutants (eSpCas9 and "high-fidelity" Cas9) by modification of different amino acids to significantly reduce off-target effects [107, 108]. While these new mutants and other recent approaches are promising, off-target activity for each gRNA should be tested carefully before use in the clinic to avoid unintended mutagenesis in other regions of the genome [109].

Research has also focused on increasing the repertoire of host sequences that can be targeted by the CRISPR/Cas system, which is dictated by the recognition of the PAM sequence by Cas9. Ideally, the PAM sequence should be within $10 \mathrm{bp}$ of the target sequence; thus, in some regions of the genome, there might be paucity of PAM sequences. Cas9 proteins of various bacterial species have different PAM motif requirements [70, 110-112], which can be naturally exploited to expand the CRISPR/Cas target space and increase the repertoire of accessible therapeutic targets. The most commonly used Cas9 is the SpCas9, which recognizes a short NGG PAM sequence, allowing it to be used across many genomic regions $[90,113]$. Other Cas9 proteins, such as those of $S$. thermophilus and Neisseria meningitides, require the PAM motifs NNAGAAW and NNNGATT, respectively. In addition, Streptococcus aureus Cas9 that recognizes a NNGRRN PAM motif has a dual interest as it is also useful for AAV delivery in vivo due to its smaller (3.1 kb versus $4.2 \mathrm{~kb}$ for SpCas9) size $[112,114]$. Along this line, the $2.9 \mathrm{~kb}$ Cas9 from Campylobacter jejuni (CjCas9) also offers an attractive option for gene delivery purposes [115]. CjCas9 has been packaged into an AAV2/9 vector along with a gRNA-targeting Vegf and delivered to the retina in a mouse model of choroidal neovascularization (CNV) [115]. This opens up the possibility that this strategy could be an alternative to repeated administration of pharmacological antiVEGF treatment for AMD. Lastly, the CRISPR-Cpf1 system identified in Acidaminococcus and Lachnospiraceae bacteria, which requires the PAM motif TTTN, has a dual advantage for genome editing because, in addition to a novel PAM 
sequence [116], it induces staggered cuts away from the critical seed region thus preventing NHEJ and increasing the efficiency of HDR [117].

To increase the repertoire of genomic target sequences even further, recent work has been aimed at artificially engineering SpCas9 and SaCas9 with alternative PAM recognition sites $[118,119]$. SpCas9 recognizing PAM target sites NGA and NGCG are known as "VQR" and "VRER", respectively; the modified SaCas9 known as "KKH" has a PAM recognition site NNNRRT. Since CRISPR/Cas9 technology was first used in 2012 for genome-editing purposes, significant advances have occurred to improve efficiency and specificity of the nucleases. The use and development of Cas9 nucleases with different PAM motifs may expand the use of CRISPR/ Cas technology throughout the human genome.

\section{In Vivo CRISPR/Cas Genome Editing}

CRISPR/Cas genome editing in animal models has been useful for developing and testing possible therapeutic techniques that could represent sight-saving approaches in the future for patients. The biggest challenge researchers face is the delivery of the CRISPR system directly into the tissue or cells of interest in the retina. As mentioned above, AAV vectors are the most effective gene delivery method for a variety of retinal cells including photoreceptors and RPE [120]. However, their limited cloning capacity has not facilitated their application as a vehicle for CRISPR/Cas. CRISPR/Cas studies with AAV have been previously explored in the field of brain diseases [121] where the delivery of the SpCas9 and gRNA was divided between two vectors. Hung and colleagues applied a similar approach to the mouse retina whereby intravitreal administration of an AAV2/2 vector mediated the delivery of a CRISPR/Cas system designed to disrupt yellow fluorescent protein expression in a Thyl-YFP transgenic mouse model [122]. This resulted in an $84 \%$ reduction of YFP expression, providing for the first time proof of concept for CRISPR/Cas genome editing in the retina in vivo.

The use of dual AAV2/8 systems for the delivery of CRISPR-Cas9 components into the retina was also used to knock out the $\mathrm{Nrl}$ (neural retina-specific leucine zipper) gene in postmitotic photoreceptors. Subretinal injection of the dual AAV system prevented cone degeneration and restored the survival of rod photoreceptors in three different genetic mouse models of retinal degeneration $\left(\mathrm{Rho}^{-1-}\right.$ mice, $\mathrm{Nrl}-\mathrm{L}$ EGFP/Rd10 mice, and in RHO P347S transgenic mice) [123]. Similarly, subretinal injection of a dual AAV2/5 CRISPR/Cas9 system in mice deleted the wild-type mouse intron 25 of the causative LCA10 gene CEP290 [106]. This intron is homologous to the human intron 26 that houses a variant, which is the most prevalent recurrent causative mutation of LCA10 $[124,125]$. Hence, this in vivo study is a proof of concept for the potential treatment of patients by ablation of the intronic variant [106]. In addition, the authors developed a self-limiting CRISPR/Cas9 system by incorporating recognition sites for the gRNAs into the SpCas9 plasmid, limiting the expression time of the Cas9. This selflimiting Cas9 approach lowers the chance of undesirable off-target events, potential toxicity, and SpCas9-specific cellular immune response [126].

The use of purified Cas9 ribonucleoproteins (RNP) has also been studied in the retina as an alternative delivery approach to AAV. This method reduces the time of Cas9 exposure potentially reducing off-targets, as the Cas9 RNP complex is degraded in the cell $24 \mathrm{~h}$ after delivery $[102,103]$. Subretinal delivery of Cas9 RNP-targeting Vegf in a mouse model of CNV significantly reduced expression [105], thus providing preliminary evidence that this method could be used for an in vivo treatment of patients with AMD and more importantly expanding the possibilities for the treatment of retinal dystrophies using purified Cas9 proteins delivered directly into the retina. Further studies are needed in order to determine if the in vivo delivery of Cas9 RNP into the retinal cells is as efficient as viral vector-mediated delivery by subretinal injections.

These above in vivo studies used CRISPR/Cas9 technology to mediate NHEJ, which results in INDELs and gene inactivation. A major problem that still remains to be addressed is how to achieve effective and accurate genome editing in the retina, as photoreceptors are postmitotic cells and largely lack HDR repair mechanisms. Suzuki and colleagues developed a novel strategy called homologyindependent targeted integration (HITI), which allows for targeted NHEJ knock-in in nondividing cells, such as the photoreceptors [127]. After subretinal injection of the AAV2/8- or 2/9-vehiculed HITI system in a rat model of $\mathrm{RP}$, correct knock-in preserved the thickness of the ONL and improved visual function. Therefore, this approach is a highly promising solution for postmitotic neurons, as it relies on the NHEJ mechanism, as opposed to HDR, for functional integration of a desired DNA sequence.

The use of CRISPR/Cas9 system and HDR in preclinical animal models has also been performed. Wu and colleagues used this technique to determine the causative variant for the RP phenotype found in the "rodless" (rd1) mouse model. The $r d l$ mice carry two homozygous variants in Pde6b: a nonsense mutation (Y347X) in exon 7 and a murine leukemia virus insertion in intron 1. Following CRISPRmediated correction of the nonsense variant, the retinal phenotype of the treated mice was restored demonstrating that the Y347X mutation was pathogenic [128]. Similarly, the pathogenicity of a novel missense variant in REEP6-causing $\mathrm{RP}$, was proven by generating a mouse knock-in model of Reep6 using CRISPR/Cas9 technology [129].

One of the biggest challenges before the CRISPR revolution was the treatment of autosomal dominant conditions, in which specific inactivation of the mutant allele is required to restore the phenotype. The treatment of these disorders was previously considered as complicated, as gene augmentation approaches did not directly target the pathogenic gene. The development of CRISPR technology has now changed the landscape of dominant disorders. One promising therapeutic approach is to decrease gene transcription through a strategy known as CRISPR interference (CRISPRi) [130]. In this strategy, Cas9 lacks nuclease activity, known as dead Cas9 (dCas9). Blockage of the transcriptional machinery occurs when dCas9 is coupled with a sequence-specific gRNA, 
preventing the RNA polymerase and transcription factors from transcribing genes. This strategy has been successfully achieved in eukaryotes and human cells [131-133]. Currently, this approach has not been applied to retinal dystrophies, but it carries a great potential due to the minimal off-target effects, which is an improvement to previous strategies involving RNA interference [134, 135].

Ablation of the mutant allele using CRISPR/Cas9 technology is another strategy that has been used in dominant forms for RP due to mutations in the gene encoding Rhodop$\sin (R H O)$. Bakondi and colleagues targeted an allele-specific PAM sequence present only in the Rho ${ }^{\$ 334}$ mutant allele of an RP mouse model. Following subretinal administration and electroporation of the CRISPR components, the photoreceptor phenotype was rescued and visual acuity increased by 53\% [136]. Similarly, Latella et al. performed a targeted knockout of a patient-derived mutant $R H O \mathrm{P} 23 \mathrm{H}$ minigene in a transgenic mouse model. Subretinal electroporation of Cas 9 and two gRNA targeting the $5^{\prime}$ and the $3^{\prime}$ regions of exon 1 resulted in reduced expression of the RHO gene [137]. These studies carry huge promise for the use of CRISPR/Cas systems to inactivate autosomal dominant pathogenic alleles in humans.

The rapid development of these technologies and the success achieved by proof-of-concept studies in vivo are speeding up the clinical translation of CRISPR technology. There is currently no CRISPR-based clinical trial for eye disease. Nonetheless, this may soon change as EDITAS medicine appears dedicated to bringing the aforementioned intron 26 skipping approach for CEP290 to LCA10 patients (https://www.allergan.com/news/news/thomson-reuters/ allergan-and-editas-medicine-enter-into-strategic).

\section{Ex Vivo Gene Correction and Cell- Based Therapy}

While gene-based therapies may halt or at least slow down the progression of the disease by targeting dysfunctional cells, another promising approach in treating retinal dystrophies is stem cell-derived retinal cell transplantation. The retina develops from the neuroectoderm, thus, like any other CNS tissue, presents a low regeneration potential. Therefore, IRDs caused by degeneration or loss of photoreceptors could potentially benefit from cell-based therapies, which would restore a functional retina and reverse the ocular condition.

The first evidence showing functional photoreceptor replacement was achieved when freshly dissociated rod photoreceptors were transplanted into the subretinal space [138]. However, the number of transplanted cells could not be increased in vitro due to their postmitotic state. Thus, there was a need to increase the number of photoreceptors for efficient transplantation into the donor retina. Lamba and colleagues showed that human embryonic stem cells (ESCs) can be directed to a retinal cell fate and differentiated into retinal precursors [139]. The transplantation of these ESCderived photoreceptors precursors into the subretinal space of an LCA mouse model resulted in restoration of the light response, establishing ESCs as a source for photoreceptor replacement [140]. ESCs present a high proliferative, selfrenewal, and differentiation potential, which makes them an ideal tool to study human diseases in vitro.

However, the use of ESCs is associated with controversial and ethical considerations, thus severely impeding major progress towards exploiting their full potential. Takahashi et al. performed groundbreaking work in 2007, which overcame the major limitations associated with the use of human ESCs. Takahashi et al. demonstrated that it is possible to generate induced pluripotent stem cells (iPSCs) from adult human fibroblasts by a reprograming process, which involves expression of four transcription factors that revert the somatic cells to a pluripotent state [141]. These cells have the potential to replace patient's tissue and represent a large source of cells for the study of human disease [142, 143]. In addition, iPSC-derived cells have two major advantages in terms of cell transplantation: they avoid the ethical issues associated with the use of embryonic or fetal tissue and they offer the possibility of autologous transplantation avoiding risks of immune rejection.

Both ESCs and iPSCs have been used extensively in the area of stem cell-derived photoreceptor generation and transplantation. Sasai and colleagues revolutionized this field by showing that it is possible to mimic optic morphogenesis in 3D culture using murine [144] and human [145] ESCs and thus obtain a large source of appropriate-staged photoreceptor precursors. It was subsequently shown that, if present in sufficient numbers, both ESC-derived and donor photoreceptor precursors could restore visual function in preclinical retinal models [140, 146-149]. In addition, it was demonstrated that photoreceptor precursors [150-152] as well as functional [153] photoreceptors could also be obtained from iPSCs. Moreover, iPSC-derived photoreceptor precursors were transplantable and could also restore vision in preclinical models [154]. Human ESCs and iPSC will continue to have a huge impact on the study and the treatment of human eye disease, as more optimal and standardized differentiation protocols continue to be developed.

The coupling of iPSC and CRISPR/Cas genomeediting technologies to repair patient-specific mutations brings us to a new era of precise and personalized medicine for patients. Advances have already been made for the CRISPR/Cas-mediated correction of pathogenic mutations causing retinal dystrophies in patient's iPSCs. Bassuk and colleagues were the first to demonstrate the potential of this approach by correcting a missense mutation in $R P G R$ responsible for X-linked RP [155]. Burnight and colleagues performed proof-of-concept studies for the correction of an exonic, deep intronic, and dominant gain of function variants: targeting an Alu insertion in exon 9 of $M A K$ restored the retinal transcript and protein, NHEJ corrected a cryptic splice variant in CEP290-causing LCA10, and mutant allele-specific targeting invalidated the dominant Pro23His mutation in the RHO gene [156]. Further upstream, the most prevalent c.2299delG mutation in the USH2A gene, responsible for Usher syndrome type 2, was corrected in patient's fibroblasts using CRISPR/Cas9 and HDR [157]. These proof-of-concept studies support the development 
of personalized iPSC-based transplantation therapies for retinal disease. On a different note, CRISPR/Cas technology in iPSCs has been used for fluorescent reporter gene knockin at the termination codon of the cone-rod homeobox $(\mathrm{Cr} x)$ gene, a photoreceptor-specific transcription factor gene. This allows the real-time monitoring of photoreceptor differentiation [158], demonstrating the interest of this technology also for fundamental research.

Following on from the big and promising advances, which demonstrated that stem cell-derived photoreceptor transplantation can restore rod- and cone-mediated vision, recent studies demonstrated that these transplanted cells do not integrate into nondegenerative host retinas. Instead, postmitotic donor and host photoreceptors engage in the transfer of cellular material, such as RNA and proteins including Rhodopsin [149, 159-161]. The visual improvements observed after stem cell-derived photoreceptor transplantation were hypothesized to be the result of endogenous photoreceptors supplemented by donor cellderived proteins. More recently, it was shown that both cell integration and cytoplasmic transfer can take place in degenerative hosts and that the relative contributions would depend on the local host environment [162]. Elucidation of the underlying mechanisms of this cellular material transfer could lead to novel therapeutic approaches in introducing functional proteins into dysfunctional photoreceptors as an alternative to gene replacement. In particular, it opens up the attractive possibility that Cas9 could be delivered as a purified protein for genome editing of viable photoreceptors.

The use of stem cell-derived photoreceptors is a powerful tool for the understanding of human retinal development and disease modeling and underlies a great potential for developing cell transplantation therapies. Such therapies are already underway in the clinic using hESC- [163-165] or hiPSC-derived [166] RPE. Initially, hESC-derived RPE was subretinally administered into AMD and Stargardt patients as dissociated cells. These cells safely persisted over time in the host retina and stably rescued visual acuity in a subset of patients [164]. Just recently, an RPE patch comprising a fully differentiated hESC-derived RPE monolayer on a coated, synthetic basement membrane was transplanted into AMD patients [165]. A one-year follow-up showed persistence of the sheet, which was associated with increased visual acuity and reading speed. It remains to be seen if these improvements will be stable over time. Lastly, the first ever, autologous transplantation for the retina was performed using a free hiPSC-derived RPE monolayer [166]. A oneyear follow-up showed that the transplantation was safe and no immune response was provoked even in the absence of immunosuppression. This provides hope for the future autologous transplantation of genome-edited retinal cells in patients. Nonetheless, further work is required to establish robust and reproducible protocols for the generation of iPSC-derived photoreceptors. In addition, if such cells are transplanted following gene mutation repair, stringent quality control of the iPSCs before and after gene correction is extremely important. Furthermore, a detailed screening for possible off-target effects triggered by CRISPR/Cas has to be performed prior to transplantation into the diseased host retina.

\section{Future Challenges and Perspectives}

The eye, more specifically the posterior retina, has proven to be a powerful model for the development of pioneer therapies, which could later be applied to other parts of the CNS. Despite the current success achieved by researchers and the relative ease and precise manipulation of the genome using the CRISPR/Cas system, improvements are being made. These are focused on the development of more efficient delivery methods, the identification and understanding of the off-target events, and increasing the efficiency of mutation correction. All these matters should be carefully addressed before this strategy can be safely applied in the clinic.

Potential delivery methods of the CRISPR/Cas components can be diverse. For an in vivo application, the ideal vehicle would be an AAV vector. The limitation of this method, in addition to size restrictions, is the constitutive expression of the Cas 9 protein in the host organism, which increases the risk of unwanted off-target events in the genome [98-100]. The use of Cas9 RNP has been shown to be effective in vivo for reducing off-target events $[101,102,167]$, although to our knowledge there has not been a study directly comparing the off-target effects of a given gRNA by AAV or RNP delivery. Thus, future research is needed in order to elucidate the most effective way, with high on-target activity and null off-target activity, to deliver CRISPR/Cas components in vivo.

A variety of methods aimed at testing for off-target mutations have been developed [168-170]. These methods are based on algorithms to computationally test homologous regions in the genome. However, currently, there is no gold standard, and it is not yet clear if Cas9 has the potential to alter other nonhomologous regions in the genome. Some studies have performed whole exome sequencing (WES) in CRISPR-treated cells and organisms [171, 172], providing an accurate and comprehensive way of testing off-target mutations. Such approaches should be taken into consideration following ex vivo gene correction in view of future transplantation into the patient.

In addition to improving the understanding of the offtarget effects created by Cas9, much effort has focused on developing methods to enhance genome-editing efficiency. In cases where gene correction is required, the HDR repair pathway is needed, and this is incompatible with postmitotic photoreceptor targets. Exciting new developments in HDR-independent base-editing strategies have shown promise for gene correction in postmitotic cells. In these cases, Cas9 is fused to a cytidine deaminase to create a base-editor tool at the specific genome target $[173,174]$, thus circumventing the need for cell division. In addition, as mentioned above, the HITI approach also carries a great promise for precise gene correction in postmitotic cells by using the NHEJ pathway [127].

Overall, the future looks bright for the use of CRISPR/ Cas genome editing in ophthalmology, and it is likely that 
the studies presented here are just the beginning of what is to come.

\section{Conflicts of Interest}

The authors declare that they have no conflicts of interest.

\section{Acknowledgments}

The authors thank M. Diakatou for critical reading of the manuscript and helpful comments. The authors also thank the patient association SOS Retinite, which funded Carla Sanjurjo-Soriano.

\section{References}

[1] A. London, I. Benhar, and M. Schwartz, "The retina as a window to the brain-from eye research to CNS disorders," Nature Reviews Neurology, vol. 9, no. 1, pp. 44-53, 2013.

[2] J. W. Streilein, "Ocular immune privilege: therapeutic opportunities from an experiment of nature," Nature Reviews Immunology, vol. 3, no. 11, pp. 879-889, 2003.

[3] I. Benhar, A. London, and M. Schwartz, "The privileged immunity of immune privileged organs: the case of the eye," Frontiers in Immunology, vol. 3, p. 296, 2012.

[4] C. Kaur, W. Foulds, and E. Ling, "Blood-retinal barrier in hypoxic ischaemic conditions: basic concepts, clinical features and management," Progress in Retinal and Eye Research, vol. 27, no. 6, pp. 622-647, 2008.

[5] L. Benowitz and Y. Yin, "Rewiring the injured CNS: lessons from the optic nerve," Experimental Neurology, vol. 209, no. 2, pp. 389-398, 2008.

[6] M. Vidal-Sanz, G. M. Bray, M. P. Villegas-Perez, S. Thanos, and A. J. Aguayo, "Axonal regeneration and synapse formation in the superior colliculus by retinal ganglion cells in the adult rat," The Journal of Neuroscience, vol. 7, no. 9, pp. 2894-2909, 1987.

[7] P. Lingor, N. Teusch, K. Schwarz et al., "Inhibition of Rho kinase (ROCK) increases neurite outgrowth on chondroitin sulphate proteoglycan in vitro and axonal regeneration in the adult optic nerve in vivo," Journal of Neurochemistry, vol. 103, no. 1, pp. 181-189, 2007.

[8] A. London, E. Itskovich, I. Benhar et al., "Neuroprotection and progenitor cell renewal in the injured adult murine retina requires healing monocyte-derived macrophages," Journal of Experimental Medicine, vol. 208, no. 1, pp. 23-39, 2011.

[9] K. Kozar, M. A. Ciemerych, V. I. Rebel et al., "Mouse development and cell proliferation in the absence of D-cyclins," Cell, vol. 118, no. 4, pp. 477-491, 2004.

[10] C. Zaverucha-Do-Valle, F. Gubert, M. Bargas-Rega et al., "Bone marrow mononuclear cells increase retinal ganglion cell survival and axon regeneration in the adult rat," Cell Transplantation, vol. 20, no. 3, pp. 391-406, 2011.

[11] R. Shechter, A. Ronen, A. Rolls et al., "Toll-like receptor 4 restricts retinal progenitor cell proliferation," Journal of Cell Biology, vol. 183, no. 3, pp. 393-400, 2008.

[12] S. Hughes, H. Yang, and T. Chan-ling, "Vascularization of the human fetal retina: roles of vasculogenesis and angiogenesis," Investigative Ophthalmology \& Visual Science, vol. 41, no. 5, pp. 1217-1228, 2000.
[13] J. Cai, K. C. Nelson, M. Wu, P. Sternberg Jr, and D. P. Jones, "Oxidative damage and protection of the RPE," Progress in Retinal and Eye Research, vol. 19, no. 2, pp. 205-221, 2000.

[14] X. Gu, N. J. Neric, J. S. Crabb et al., "Age-related changes in the retinal pigment epithelium (RPE)," PLoS One, vol. 7, no. 6, article e38673, 2012.

[15] M. A. Dyer and C. L. Cepko, "Regulating proliferation during retinal development," Nature Reviews Neuroscience, vol. 2, no. 5, pp. 333-342, 2001.

[16] W. Berger, B. Kloeckener-Gruissem, and J. Neidhardt, "The molecular basis of human retinal and vitreoretinal diseases," Progress in Retinal and Eye Research, vol. 29, no. 5, pp. 335-375, 2010.

[17] D. T. Hartong, E. L. Berson, and T. P. Dryja, "Retinitis pigmentosa," The Lancet, vol. 368, no. 9549, pp. 1795-1809, 2006.

[18] M. M. Sohocki, S. P. Daiger, S. J. Bowne et al., "Prevalence of mutations causing retinitis pigmentosa and other inherited retinopathies," Human Mutation, vol. 17, no. 1, pp. 42-51, 2001.

[19] L. S. Sullivan and S. P. Daiger, "Inherited retinal degeneration: exceptional genetic and clinical heterogeneity," Molecular Medicine Today, vol. 2, no. 9, pp. 380-386, 1996.

[20] P. Tanna, R. W. Strauss, K. Fujinami, and M. Michaelides, "Stargardt disease: clinical features, molecular genetics, animal models and therapeutic options," British Journal of Ophthalmology, vol. 101, no. 1, pp. 25-30, 2017.

[21] S. K. Verbakel, R. A. C. van Huet, C. J. F. Boon et al., "Nonsyndromic retinitis pigmentosa," Progress in Retinal and Eye Research, 2018.

[22] C. P. Hamel, "Cone rod dystrophies," Orphanet Journal of Rare Diseases, vol. 2, no. 1, p. 7, 2007.

[23] H. Kremer, E. van Wijk, T. Märker, U. Wolfrum, and R. Roepman, "Usher syndrome: molecular links of pathogenesis, proteins and pathways," Human Molecular Genetics, vol. 15, Supplement 2, pp. R262-R270, 2006.

[24] J. D. Sengillo, S. Justus, Y. T. Tsai, T. Cabral, and S. H. Tsang, "Gene and cell-based therapies for inherited retinal disorders: an update," American Journal of Medical Genetics Part C: Seminars in Medical Genetics, vol. 172, no. 4, pp. 349-366, 2016.

[25] K. H. Warrington Jr. and R. W. Herzog, "Treatment of human disease by adeno-associated viral gene transfer," Human Genetics, vol. 119, no. 6, pp. 571-603, 2006.

[26] S. Daya and K. I. Berns, "Gene therapy using adenoassociated virus vectors," Clinical Microbiology Reviews, vol. 21, no. 4, pp. 583-593, 2008.

[27] M. K. Lin, Y.-T. Tsai, and S. H. Tsang, "Emerging treatments for retinitis pigmentosa: genes and stem cells, as well as new electronic and medical therapies, are gaining ground," Retinal Physician, vol. 12, pp. 52-70, 2015.

[28] P. Yu-Wai-Man, "Genetic manipulation for inherited neurodegenerative diseases: myth or reality?," British Journal of Ophthalmology, vol. 100, no. 10, pp. 1322-1331, 2016.

[29] G. M. Acland, G. D. Aguirre, J. Ray et al., "Gene therapy restores vision in a canine model of childhood blindness," Nature Genetics, vol. 28, no. 1, pp. 92-95, 2001.

[30] J. W. B. Bainbridge, A. J. Smith, S. S. Barker et al., "Effect of gene therapy on visual function in Leber's congenital amaurosis," The New England Journal of Medicine, vol. 358, no. 21, pp. 2231-2239, 2008. 
[31] A. M. Maguire, F. Simonelli, E. A. Pierce et al., "Safety and efficacy of gene transfer for Leber's congenital amaurosis," The New England Journal of Medicine, vol. 358, no. 21, pp. 2240-2248, 2008.

[32] A. V. Cideciyan, T. S. Aleman, S. L. Boye et al., "Human gene therapy for RPE65 isomerase deficiency activates the retinoid cycle of vision but with slow rod kinetics," Proceedings of the National Academy of Sciences of the United States of America, vol. 105, no. 39, pp. 15112-15117, 2008.

[33] J. Bennett, J. Wellman, K. A. Marshall et al., "Safety and durability of effect of contralateral-eye administration of AAV2 gene therapy in patients with childhood-onset blindness caused by RPE65 mutations: a follow-on phase 1 trial," The Lancet, vol. 388, no. 10045, pp. 661-672, 2016.

[34] W. W. Hauswirth, T. S. Aleman, S. Kaushal et al., "Treatment of Leber congenital amaurosis due to RPE65 mutations by ocular subretinal injection of adeno-associated virus gene vector: short-term results of a phase I trial," Human Gene Therapy, vol. 19, no. 10, pp. 979-990, 2008.

[35] S. Russell, J. Bennett, J. A. Wellman et al., "Efficacy and safety of voretigene neparvovec (AAV2-hRPE65v2) in patients with $R P E 65$-mediated inherited retinal dystrophy: a randomised, controlled, open-label, phase 3 trial," The Lancet, vol. 390, no. 10097, pp. 849-860, 2017.

[36] R. E. MacLaren, M. Groppe, A. R. Barnard et al., "Retinal gene therapy in patients with choroideremia: initial findings from a phase $1 / 2$ clinical trial," The Lancet, vol. 383, no. 9923, pp. 1129-1137, 2014.

[37] I. S. Dimopoulos, S. Chan, R. E. MacLaren, and I. M. MacDonald, "Pathogenic mechanisms and the prospect of gene therapy for choroideremia," Expert Opinion on Orphan Drugs, vol. 3, no. 7, pp. 787-798, 2015.

[38] T. Tolmachova, O. E. Tolmachov, A. R. Barnard et al., "Functional expression of Rab escort protein 1 following AAV2mediated gene delivery in the retina of choroideremia mice and human cells ex vivo," Journal of Molecular Medicine, vol. 91, no. 7, pp. 825-837, 2013.

[39] N. Cereso, M. O. Pequignot, L. Robert et al., "Proof of concept for AAV2/5-mediated gene therapy in iPSC-derived retinal pigment epithelium of a choroideremia patient," Molecular Therapy Methods \& Clinical Development, vol. 1, pp. 14011-14013, 2014.

[40] A. Black, V. Vasireddy, D. C. Chung et al., "Adeno-associated virus 8-mediated gene therapy for choroideremia: preclinical studies in in vitro and in vivo models," The Journal of Gene Medicine, vol. 16, no. 5-6, pp. 122-130, 2014.

[41] N. G. Ghazi, E. B. Abboud, S. R. Nowilaty et al., "Treatment of retinitis pigmentosa due to MERTK mutations by ocular subretinal injection of adeno-associated virus gene vector: results of a phase I trial," Human Genetics, vol. 135, no. 3, pp. 327343, 2016.

[42] P. L. Hermonat, J. G. Quirk, B. M. Bishop, and L. Han, “The packaging capacity of adeno-associated virus (AAV) and the potential for wild-type-plus AAV gene therapy vectors," FEBS Letters, vol. 407, no. 1, pp. 78-84, 1997.

[43] Z. Wu, H. Yang, and P. Colosi, "Effect of genome size on AAV vector packaging," Molecular Therapy, vol. 18, no. 1, pp. 80-86, 2010.

[44] Y. Wang, C. Ling, L. Song et al., "Limitations of encapsidation of recombinant self-complementary adeno-associated viral genomes in different serotype capsids and their quantitation,"
Human Gene Therapy Methods, vol. 23, no. 4, pp. 225-233, 2012.

[45] J. Kong, S. R. Kim, K. Binley et al., "Correction of the disease phenotype in the mouse model of Stargardt disease by lentiviral gene therapy," Gene Therapy, vol. 15, no. 19, pp. 1311-1320, 2008.

[46] K. Binley, P. Widdowson, J. Loader et al., "Transduction of photoreceptors with equine infectious anemia virus lentiviral vectors: safety and biodistribution of StarGen for Stargardt disease," Investigative Opthalmology \& Visual Science, vol. 54, no. 6, pp. 4061-4071, 2013.

[47] D. S. Williams and V. S. Lopes, "The many different cellular functions of MYO7A in the retina," Biochemical Society Transactions, vol. 39, no. 5, pp. 1207-1210, 2011.

[48] M. Zallocchi, K. Binley, Y. Lad et al., "EIAV-based retinal gene therapy in the shaker1 mouse model for Usher syndrome type 1B: development of UshStat," PLoS One, vol. 9, no. 4, article e94272, 2014.

[49] A. V. Cideciyan, S. G. Jacobson, W. A. Beltran et al., "Human retinal gene therapy for Leber congenital amaurosis shows advancing retinal degeneration despite enduring visual improvement," Proceedings of the National Academy of Sciences of the United States of America, vol. 110, no. 6, pp. E517-E525, 2013.

[50] S. G. Jacobson, A. V. Cideciyan, A. J. Roman et al., "Improvement and decline in vision with gene therapy in childhood blindness," The New England Journal of Medicine, vol. 372, no. 20, pp. 1920-1926, 2015.

[51] R. J. Davis, C. W. Hsu, Y. T. Tsai et al., "Therapeutic margins in a novel preclinical model of retinitis pigmentosa," The Journal of Neuroscience, vol. 33, no. 33, pp. 13475-13483, 2013.

[52] K. J. Wert, J. Sancho-Pelluz, and S. H. Tsang, "Mid-stage intervention achieves similar efficacy as conventional earlystage treatment using gene therapy in a pre-clinical model of retinitis pigmentosa," Human Molecular Genetics, vol. 23, no. 2, pp. 514-523, 2014.

[53] J. B. Hurley and J. R. Chao, "It's never too late to save a photoreceptor," The Journal of Clinical Investigation, vol. 125, no. 9, pp. 3424-3426, 2015.

[54] C. L. Cepko and L. H. Vandenberghe, "Retinal gene therapy coming of age," Human Gene Therapy, vol. 24, no. 3, pp. 242-244, 2013.

[55] A. M. Maguire, K. A. High, A. Auricchio et al., "Age-dependent effects of RPE65 gene therapy for Leber's congenital amaurosis: a phase 1 dose-escalation trial," The Lancet, vol. 374, no. 9701, pp. 1597-1605, 2009.

[56] J. W. B. Bainbridge, M. S. Mehat, V. Sundaram et al., "Longterm effect of gene therapy on Leber's congenital amaurosis," The New England Journal of Medicine, vol. 372, no. 20, pp. 1887-1897, 2015.

[57] G. S. Yang, M. Schmidt, Z. Yan et al., "Virus-mediated transduction of murine retina with adeno-associated virus: effects of viral capsid and genome size," Journal of Virology, vol. 76, no. 15, pp. 7651-7660, 2002.

[58] L. H. Vandenberghe and A. Auricchio, "Novel adenoassociated viral vectors for retinal gene therapy," Gene Ther$a p y$, vol. 19, no. 2, pp. 162-168, 2012.

[59] F. M. Mowat, K. R. Gornik, A. Dinculescu et al., "Tyrosine capsid-mutant AAV vectors for gene delivery to the canine 
retina from a subretinal or intravitreal approach," Gene Therapy, vol. 21, no. 1, pp. 96-105, 2014.

[60] A. Georgiadis, Y. Duran, J. Ribeiro et al., "Development of an optimized AAV2/5 gene therapy vector for Leber congenital amaurosis owing to defects in RPE65," Gene Therapy, vol. 23, no. 12, pp. 857-862, 2016.

[61] M. D. Fischer, M. E. McClements, C. Martinez-Fernandez de la Camara et al., "Codon-optimized RPGR improves stability and efficacy of AAV8 gene therapy in two mouse models of X-linked retinitis pigmentosa," Molecular Therapy, vol. 25, no. 8, pp. 1854-1865, 2017.

[62] G. J. Farrar, S. Millington-Ward, N. Chadderton, P. Humphries, and P. F. Kenna, "Gene-based therapies for dominantly inherited retinopathies," Gene Therapy, vol. 19, no. 2, pp. 137-144, 2012.

[63] A. Palfi, M. Ader, A. S. Kiang et al., "RNAi-based suppression and replacement of $r d s$-peripherin in retinal organotypic culture," Human Mutation, vol. 27, no. 3, pp. 260-268, 2006.

[64] M. O'Reilly, A. Palfi, N. Chadderton et al., "RNA interference-mediated suppression and replacement of human rhodopsin in vivo," The American Journal of Human Genetics, vol. 81, no. 1, pp. 127-135, 2007.

[65] N. Chadderton, S. Millington-Ward, A. Palfi et al., "Improved retinal function in a mouse model of dominant retinitis pigmentosa following AAV-delivered gene therapy," Molecular Therapy, vol. 17, no. 4, pp. 593-599, 2009.

[66] S. Millington-Ward, N. Chadderton, M. O'Reilly et al., "Suppression and replacement gene therapy for autosomal dominant disease in a murine model of dominant retinitis pigmentosa," Molecular Therapy, vol. 19, no. 4, pp. 642649, 2011.

[67] B. Pardo, B. Gómez-González, and A. Aguilera, "DNA repair in mammalian cells: DNA double-strand break repair: how to fix a broken relationship," Cellular and Molecular Life Sciences, vol. 66, no. 6, pp. 1039-1056, 2009.

[68] M. Yanik, B. Müller, F. Song et al., "In vivo genome editing as a potential treatment strategy for inherited retinal dystrophies," Progress in Retinal and Eye Research, vol. 56, pp. 1-18, 2017.

[69] M. Christian, T. Cermak, E. L. Doyle et al., "Targeting DNA double-strand breaks with TAL effector nucleases," Genetics, vol. 186, no. 2, pp. 757-761, 2010.

[70] L. Cong, F. A. Ran, D. Cox et al., "Multiplex genome engineering using CRISPR/Cas systems," Science, vol. 339, no. 6121, pp. 819-823, 2013.

[71] W.-D. Heyer, K. T. Ehmsen, and J. Liu, "Regulation of homologous recombination in eukaryotes," Annual Review of Genetics, vol. 44, no. 1, pp. 113-139, 2010.

[72] B. Schierling, N. Dannemann, L. Gabsalilow, W. Wende, T. Cathomen, and A. Pingoud, "A novel zinc-finger nuclease platform with a sequence-specific cleavage module," Nucleic Acids Research, vol. 40, no. 6, pp. 2623-2638, 2012.

[73] S. A. Wolfe, L. Nekludova, and C. O. Pabo, "DNA recognition by Cys2His2 zinc finger proteins," Annual Review of Biophysics and Biomolecular Structure, vol. 29, no. 1, pp. 183-212, 2000.

[74] Y. G. Kim, J. Cha, and S. Chandrasegaran, "Hybrid restriction enzymes: zinc finger fusions to Fok I cleavage domain," Proceedings of the National Academy of Sciences of the United States of America, vol. 93, no. 3, pp. 1156-1160, 1996.
[75] Y. G. Kim, Y. Shi, J. M. Berg, and S. Chandrasegaran, "Sitespecific cleavage of DNA-RNA hybrids by zinc finger/FokI cleavage domain fusions," Gene, vol. 203, no. 1, pp. 43-49, 1997.

[76] J. Smith, M. Bibikova, F. G. Whitby, A. R. Reddy, S. Chandrasegaran, and D. Carroll, "Requirements for double-strand cleavage by chimeric restriction enzymes with zinc finger DNA-recognition domains," Nucleic Acids Research, vol. 28, no. 17, pp. 3361-3369, 2000.

[77] A. F. Gilles and M. Averof, "Functional genetics for all: engineered nucleases, CRISPR and the gene editing revolution," EvoDevo, vol. 5, no. 1, p. 43, 2014.

[78] D. Carroll, "Genome engineering with targetable nucleases," Annual Review of Biochemistry, vol. 83, no. 1, pp. 409-439, 2014.

[79] T. Gaj, C. A. Gersbach, and C. F. Barbas III, "ZFN, TALEN, and CRISPR/Cas-based methods for genome engineering," Trends in Biotechnology, vol. 31, no. 7, pp. 397-405, 2013.

[80] D. L. Greenwald, S. M. Cashman, and R. Kumar-Singh, "Engineered zinc finger nuclease-mediated homologous recombination of the human rhodopsin gene," Investigative Ophthalmology \& Visual Science, vol. 51, no. 12, pp. 63746380, 2010.

[81] N. Overlack, T. Goldmann, U. Wolfrum, and K. Nagel-Wolfrum, "Gene repair of an Usher syndrome causing mutation by zinc-finger nuclease mediated homologous recombination," Investigative Ophthalmology \& Visual Science, vol. 53, no. 7, pp. 4140-4146, 2012.

[82] B. E. Low, M. P. Krebs, J. K. Joung, S. Q. Tsai, P. M. Nishina, and M. V. Wiles, "Correction of the $\mathrm{Crb}^{1}{ }^{\text {rd8 }}$ allele and retinal phenotype in C57BL/6N mice via TALEN-mediated homology-directed repair," Investigative Ophthalmology \& Visual Science, vol. 55, no. 1, pp. 387-395, 2014.

[83] Y. Ishino, H. Shinagawa, K. Makino, M. Amemura, and A. Nakata, "Nucleotide sequence of the iap gene, responsible for alkaline phosphatase isozyme conversion in Escherichia coli, and identification of the gene product," Journal of Bacteriology, vol. 169, no. 12, pp. 5429-5433, 1987.

[84] F. J. M. Mojica, C. Ferrer, G. Juez, and F. Rodriguez-Valera, "Long stretches of short tandem repeats are present in the largest replicons of the Archaea Haloferax mediterranei and Haloferax volcanii and could be involved in replicon partitioning," Molecular Microbiology, vol. 17, no. 1, pp. 85-93, 1995.

[85] H. P. Klenk, R. A. Clayton, J. F. Tomb et al., “The complete genome sequence of the hyperthermophilic, sulphatereducing archaeon Archaeoglobus fulgidus," Nature, vol. 390, no. 6658, pp. 364-370, 1997.

[86] K. E. Nelson, R. A. Clayton, S. R. Gill et al., "Evidence for lateral gene transfer between Archaea and Bacteria from genome sequence of Thermotoga maritima," Nature, vol. 399, no. 6734, pp. 323-329, 1999.

[87] R. Jansen, J. D. A. v. Embden, W. Gaastra, and L. M. Schouls, "Identification of genes that are associated with DNA repeats in prokaryotes," Molecular Microbiology, vol. 43, no. 6, pp. 1565-1575, 2002.

[88] F. J. M. Mojica, C. Diez-Villasenor, E. Soria, and G. Juez, "Biological significance of a family of regularly spaced repeats in the genomes of Archaea, Bacteria and mitochondria," Molecular Microbiology, vol. 36, no. 1, pp. 244-246, 2000. 
[89] R. Barrangou, C. Fremaux, H. Deveau et al., "CRISPR provides acquired resistance against viruses in prokaryotes," Science, vol. 315, no. 5819, pp. 1709-1712, 2007.

[90] M. Jinek, K. Chylinski, I. Fonfara, M. Hauer, J. A. Doudna, and E. Charpentier, "A programmable dual-RNA-guided DNA endonuclease in adaptive bacterial immunity," Science, vol. 337, no. 6096, pp. 816-821, 2012.

[91] J. A. Doudna and E. Charpentier, "The new frontier of genome engineering with CRISPR-Cas9," Science, vol. 346, no. 6213, article 1258096, 2014.

[92] F. A. Ran, P. D. Hsu, C. Y. Lin et al., "Double nicking by RNA-guided CRISPR cas9 for enhanced genome editing specificity," Cell, vol. 154, no. 6, pp. 1380-1389, 2013.

[93] P. Mali, L. Yang, K. M. Esvelt et al., "RNA-guided human genome engineering via Cas9," Science, vol. 339, no. 6121, pp. 823-826, 2013.

[94] N. Chang, C. Sun, L. Gao et al., "Genome editing with RNAguided Cas9 nuclease in zebrafish embryos," Cell Research, vol. 23, no. 4, pp. 465-472, 2013.

[95] A. E. Friedland, Y. B. Tzur, K. M. Esvelt, M. P. Colaiácovo, G. M. Church, and J. A. Calarco, "Heritable genome editing in C. elegans via a CRISPR-Cas9 system," Nature Methods, vol. 10, no. 8, pp. 741-743, 2013.

[96] W. Jiang, H. Zhou, H. Bi, M. Fromm, B. Yang, and D. P. Weeks, "Demonstration of CRISPR/Cas9/sgRNA-mediated targeted gene modification in Arabidopsis, tobacco, sorghum and rice," Nucleic Acids Research, vol. 41, no. 20, article e188, 2013.

[97] J. E. DiCarlo, J. E. Norville, P. Mali, X. Rios, J. Aach, and G. M. Church, "Genome engineering in Saccharomyces cerevisiae using CRISPR-Cas systems," Nucleic Acids Research, vol. 41, no. 7, pp. 4336-4343, 2013.

[98] Y. Fu, J. A. Foden, C. Khayter et al., "High-frequency offtarget mutagenesis induced by CRISPR-Cas nucleases in human cells," Nature Biotechnology, vol. 31, no. 9, pp. 822826, 2013.

[99] P. D. Hsu, D. A. Scott, J. A. Weinstein et al., "DNA targeting specificity of RNA-guided Cas9 nucleases," Nature Biotechnology, vol. 31, no. 9, pp. 827-832, 2013.

[100] V. Pattanayak, S. Lin, J. P. Guilinger, E. Ma, J. A. Doudna, and D. R. Liu, "High-throughput profiling of off-target DNA cleavage reveals RNA-programmed Cas9 nuclease specificity," Nature Biotechnology, vol. 31, no. 9, pp. 839843, 2013.

[101] S. W. Cho, J. Lee, D. Carroll, J. S. Kim, and J. Lee, "Heritable gene knockout in Caenorhabditis elegans by direct injection of Cas9-sgRNA ribonucleoproteins," Genetics, vol. 195, no. 3, pp. 1177-1180, 2013.

[102] S. Kim, D. Kim, S. W. Cho, J. Kim, and J. S. Kim, "Highly efficient RNA-guided genome editing in human cells via delivery of purified Cas9 ribonucleoproteins," Genome Research, vol. 24, no. 6, pp. 1012-1019, 2014.

[103] S. Lin, B. T. Staahl, R. K. Alla, and J. A. Doudna, "Enhanced homology-directed human genome engineering by controlled timing of CRISPR/Cas9 delivery," eLife, vol. 3, article e04766, 2014.

[104] X. Liang, J. Potter, S. Kumar, N. Ravinder, and J. D. Chesnut, "Enhanced CRISPR/Cas9-mediated precise genome editing by improved design and delivery of gRNA, Cas9 nuclease, and donor DNA," Journal of Biotechnology, vol. 241, pp. 136-146, 2017.
[105] K. Kim, S. W. Park, J. H. Kim et al., "Genome surgery using Cas9 ribonucleoproteins for the treatment of age-related macular degeneration," Genome Research, vol. 27, no. 3, pp. 419-426, 2017.

[106] G.-X. Ruan, E. Barry, D. Yu, M. Lukason, S. H. Cheng, and A. Scaria, "CRISPR/Cas9-mediated genome editing as a therapeutic approach for Leber congenital amaurosis 10," Molecular Therapy, vol. 25, no. 2, pp. 331-341, 2017.

[107] I. M. Slaymaker, L. Gao, B. Zetsche, D. A. Scott, W. X. Yan, and F. Zhang, "Rationally engineered Cas9 nucleases with improved specificity," Science, vol. 351, no. 6268, pp. 84-88, 2016.

[108] B. P. Kleinstiver, V. Pattanayak, M. S. Prew et al., "High-fidelity CRISPR-Cas9 nucleases with no detectable genome-wide off-target effects," Nature, vol. 529, no. 7587, pp. 490-495, 2016.

[109] S. Q. Tsai, Z. Zheng, N. T. Nguyen et al., "GUIDE-seq enables genome-wide profiling of off-target cleavage by CRISPR-Cas nucleases," Nature Biotechnology, vol. 33, no. 2, pp. 187-197, 2015.

[110] K. M. Esvelt, P. Mali, J. L. Braff, M. Moosburner, S. J. Yaung, and G. M. Church, "Orthogonal Cas9 proteins for RNAguided gene regulation and editing," Nature Methods, vol. 10, no. 11, pp. 1116-1121, 2013.

[111] I. Fonfara, A. le Rhun, K. Chylinski et al., "Phylogeny of Cas9 determines functional exchangeability of dual-RNA and Cas9 among orthologous type II CRISPR-Cas systems," Nucleic Acids Research, vol. 42, no. 4, pp. 2577-2590, 2014.

[112] F. A. Ran, L. Cong, W. X. Yan et al., "In vivo genome editing using Staphylococcus aureus Cas9," Nature, vol. 520, no. 7546, pp. 186-191, 2015.

[113] W. Jiang, D. Bikard, D. Cox, F. Zhang, and L. A. Marraffini, "RNA-guided editing of bacterial genomes using CRISPRCas systems," Nature Biotechnology, vol. 31, no. 3, pp. 233239, 2013.

[114] A. E. Friedland, R. Baral, P. Singhal et al., "Characterization of Staphylococcus aureus Cas9: a smaller Cas9 for all-in-one adeno-associated virus delivery and paired nickase applications," Genome Biology, vol. 16, no. 1, p. 257, 2015.

[115] E. Kim, T. Koo, S. W. Park et al., "In vivo genome editing with a small Cas9 orthologue derived from Campylobacter jejuni," Nature Communications, vol. 8, article 14500, 2017.

[116] B. Zetsche, J. S. Gootenberg, O. O. Abudayyeh et al., "Cpf1 is a single RNA-guided endonuclease of a class 2 CRISPR-Cas system," Cell, vol. 163, no. 3, pp. 759-771, 2015.

[117] R. D. Fagerlund, R. H. J. Staals, and P. C. Fineran, "The Cpf1 CRISPR-Cas protein expands genome-editing tools," Genome Biology, vol. 16, no. 1, pp. 251-253, 2015.

[118] B. P. Kleinstiver, M. S. Prew, S. Q. Tsai et al., "Broadening the targeting range of Staphylococcus aureus CRISPR-Cas9 by modifying PAM recognition," Nature Biotechnology, vol. 33, no. 12, pp. 1293-1298, 2015.

[119] B. P. Kleinstiver, M. S. Prew, S. Q. Tsai et al., "Engineered CRISPR-Cas9 nucleases with altered PAM specificities," Nature, vol. 523, no. 7561, pp. 481-485, 2015.

[120] T. P. Day, L. C. Byrne, D. V. Schaffer, and J. G. Flannery, "Advances in AAV vector development for gene therapy in the retina," Advances in Experimental Medicine and Biology, vol. 801, pp. 687-693, 2014

[121] L. Swiech, M. Heidenreich, A. Banerjee et al., "In vivo interrogation of gene function in the mammalian brain 
using CRISPR-Cas9," Nature Biotechnology, vol. 33, no. 1, pp. 102-106, 2015.

[122] S. S. C. Hung, V. Chrysostomou, F. Li et al., "AAV-mediated CRISPR/Cas gene editing of retinal cells in vivo," Investigative Ophthalmology \& Visual Science, vol. 57, no. 7, pp. 3470-3476, 2016.

[123] W. Yu, S. Mookherjee, V. Chaitankar et al., "Nrl knockdown by AAV-delivered CRISPR/Cas9 prevents retinal degeneration in mice," Nature Communications, vol. 8, article 14716, 2017.

[124] A. I. den Hollander, R. K. Koenekoop, S. Yzer et al., "Mutations in the CEP290 (NPHP6) gene are a frequent cause of Leber congenital amaurosis," The American Journal of Human Genetics, vol. 79, no. 3, pp. 556-561, 2006.

[125] I. Perrault, N. Delphin, S. Hanein et al., "Spectrum of NPHP6/CEP290 mutations in Leber congenital amaurosis and delineation of the associated phenotype," Human Mutation, vol. 28, no. 4, p. 416, 2007.

[126] D. Wang, H. Mou, S. Li et al., "Adenovirus-mediated somatic genome editing of Pten by CRISPR/Cas9 in mouse liver in spite of Cas9-specific immune responses," Human Gene Therapy, vol. 26, no. 7, pp. 432-442, 2015.

[127] K. Suzuki, Y. Tsunekawa, R. Hernandez-Benitez et al., "In vivo genome editing via CRISPR/Cas9 mediated homologyindependent targeted integration," Nature, vol. 540, no. 7631, pp. 144-149, 2016.

[128] W.-H. Wu, Y. T. Tsai, S. Justus et al., "CRISPR repair reveals causative mutation in a preclinical model of retinitis pigmentosa," Molecular Therapy, vol. 24, no. 8, pp. 1388-1394, 2016.

[129] G. Arno, S. A. Agrawal, A. Eblimit et al., "Mutations in REEP6 cause autosomal-recessive retinitis pigmentosa," The American Journal of Human Genetics, vol. 99, no. 6, pp. 1305-1315, 2016.

[130] L. S. Qi, M. H. Larson, L. A. Gilbert et al., "Repurposing CRISPR as an RNA-guided platform for sequence-specific control of gene expression," Cell, vol. 152, no. 5, pp. 11731183, 2013.

[131] L. A. Gilbert, M. H. Larson, L. Morsut et al., "CRISPR-mediated modular RNA-guided regulation of transcription in eukaryotes," Cell, vol. 154, no. 2, pp. 442-451, 2013.

[132] M. L. Maeder, S. J. Linder, V. M. Cascio, Y. Fu, Q. H. Ho, and J. K. Joung, "CRISPR RNA-guided activation of endogenous human genes," Nature Methods, vol. 10, no. 10, pp. 977-979, 2013.

[133] L. A. Gilbert, M. A. Horlbeck, B. Adamson et al., "Genomescale CRISPR-mediated control of gene repression and activation," Cell, vol. 159, no. 3, pp. 647-661, 2014.

[134] L. Jiang, T. Z. Li, S. E. Boye, W. W. Hauswirth, J. M. Frederick, and W. Baehr, "RNAi-mediated gene suppression in a GCAP1(L151F) cone-rod dystrophy mouse model," PLoS One, vol. 8, no. 3, article e57676, 2013.

[135] S. M. Cashman, E. A. Binkley, and R. Kumar-Singh, "Towards mutation-independent silencing of genes involved in retinal degeneration by RNA interference," Gene Therapy, vol. 12, no. 15, pp. 1223-1228, 2005.

[136] B. Bakondi, W. Lv, B. Lu et al., "In vivo CRISPR/Cas9 gene editing corrects retinal dystrophy in the S334ter-3 rat model of autosomal dominant retinitis pigmentosa," Molecular Therapy, vol. 24, no. 3, pp. 556-563, 2016.

[137] M. C. Latella, M. T. di Salvo, F. Cocchiarella et al., "In vivo editing of the human mutant Rhodopsin gene by electroporation of plasmid-based CRISPR/Cas9 in the mouse retina," Molecular Therapy Nucleic Acids, vol. 5, no. 11, article e389, 2016.

[138] R. E. MacLaren, R. A. Pearson, A. MacNeil et al., "Retinal repair by transplantation of photoreceptor precursors," Nature, vol. 444, no. 7116, pp. 203-207, 2006.

[139] D. A. Lamba, M. O. Karl, C. B. Ware, and T. A. Reh, "Efficient generation of retinal progenitor cells from human embryonic stem cells," Proceedings of the National Academy of Sciences of the United States of America, vol. 103, no. 34, pp. 1276912774, 2006.

[140] D. A. Lamba, J. Gust, and T. A. Reh, "Transplantation of human embryonic stem cell-derived photoreceptors restores some visual function in Crx-deficient mice," Cell Stem Cell, vol. 4, no. 1, pp. 73-79, 2009.

[141] K. Takahashi, K. Tanabe, M. Ohnuki et al., "Induction of pluripotent stem cells from adult human fibroblasts by defined factors," Cell, vol. 131, no. 5, pp. 861-872, 2007.

[142] S. S. C. Hung, S. Khan, C. Y. Lo, A. W. Hewitt, and R. C. B. Wong, "Drug discovery using induced pluripotent stem cell models of neurodegenerative and ocular diseases," Pharmacology \& Therapeutics, vol. 177, pp. 32-43, 2017.

[143] L. A. Wiley, E. R. Burnight, A. E. Songstad et al., "Patient-specific induced pluripotent stem cells (iPSCs) for the study and treatment of retinal degenerative diseases," Progress in Retinal and Eye Research, vol. 44, pp. 15-35, 2015.

[144] M. Eiraku, N. Takata, H. Ishibashi et al., "Self-organizing optic-cup morphogenesis in three-dimensional culture," Nature, vol. 472, no. 7341, pp. 51-56, 2011.

[145] T. Nakano, S. Ando, N. Takata et al., "Self-formation of optic cups and storable stratified neural retina from human ESCs," Cell Stem Cell, vol. 10, no. 6, pp. 771-785, 2012.

[146] R. A. Pearson, A. C. Barber, M. Rizzi et al., "Restoration of vision after transplantation of photoreceptors," Nature, vol. 485, no. 7396, pp. 99-103, 2012.

[147] E. L. West, A. Gonzalez-Cordero, C. Hippert et al., "Defining the integration capacity of embryonic stem cell-derived photoreceptor precursors," Stem Cells, vol. 30, no. 7, pp. 1424$1435,2012$.

[148] A. C. Barber, C. Hippert, Y. Duran et al., "Repair of the degenerate retina by photoreceptor transplantation," Proceedings of the National Academy of Sciences of the United States of America, vol. 110, no. 1, pp. 354-359, 2013.

[149] M. S. Singh, J. Balmer, A. R. Barnard et al., “Transplanted photoreceptor precursors transfer proteins to host photoreceptors by a mechanism of cytoplasmic fusion," Nature Communications, vol. 7, article 13537, 2016.

[150] Y. Hirami, F. Osakada, K. Takahashi et al., "Generation of retinal cells from mouse and human induced pluripotent stem cells," Neuroscience Letters, vol. 458, no. 3, pp. 126131, 2009.

[151] J. S. Meyer, R. L. Shearer, E. E. Capowski et al., "Modeling early retinal development with human embryonic and induced pluripotent stem cells," Proceedings of the National Academy of Sciences of the United States of America, vol. 106, no. 39, pp. 16698-16703, 2009.

[152] S. Reichman, A. Terray, A. Slembrouck et al., "From confluent human iPS cells to self-forming neural retina and retinal pigmented epithelium," Proceedings of the National Academy of Sciences of the United States of America, vol. 111, no. 23, pp. 8518-8523, 2014. 
[153] X. Zhong, C. Gutierrez, T. Xue et al., "Generation of threedimensional retinal tissue with functional photoreceptors from human iPSCs," Nature Communications, vol. 5, p. 4047, 2014.

[154] B. A. Tucker, I. H. Park, S. D. Qi et al., "Transplantation of adult mouse iPS cell-derived photoreceptor precursors restores retinal structure and function in degenerative mice," PLoS One, vol. 6, no. 4, article e18992, 2011.

[155] A. G. Bassuk, A. Zheng, Y. Li, S. H. Tsang, and V. B. Mahajan, "Precision medicine: genetic repair of retinitis pigmentosa in patient-derived stem cells," Scientific Reports, vol. 6, no. 1, article 19969, 2016.

[156] E. R. Burnight, M. Gupta, L. A. Wiley et al., "Using CRISPRCas9 to generate gene-corrected autologous iPSCs for the treatment of inherited retinal degeneration," Molecular Therapy, vol. 25, no. 9, pp. 1999-2013, 2017.

[157] C. Fuster-García, G. García-García, E. González-Romero et al., "USH2A gene editing using the CRISPR system," Molecular Therapy Nucleic Acids, vol. 8, pp. 529-541, 2017.

[158] K. Homma, S. Usui, and M. Kaneda, "Knock-in strategy at 3 ' -end of Crx gene by CRISPR/Cas9 system shows the gene expression profiles during human photoreceptor differentiation," Genes to Cells, vol. 22, no. 3, pp. 250-264, 2017.

[159] R. A. Pearson, A. Gonzalez-Cordero, E. L. West et al., "Donor and host photoreceptors engage in material transfer following transplantation of post-mitotic photoreceptor precursors," Nature Communications, vol. 7, article 13029, 2016.

[160] T. Santos-Ferreira, S. Llonch, O. Borsch, K. Postel, J. Haas, and M. Ader, "Retinal transplantation of photoreceptors results in donor-host cytoplasmic exchange," Nature Communications, vol. 7, article 13028, 2016.

[161] S. Decembrini, C. Martin, F. Sennlaub et al., "Cone genesis tracing by the Chrnb4-EGFP mouse line: evidences of cellular material fusion after cone precursor transplantation," Molecular Therapy, vol. 25, no. 3, pp. 634-653, 2017.

[162] P. V. Waldron, F. di Marco, K. Kruczek et al., "Transplanted donor- or stem cell-derived cone photoreceptors can both integrate and undergo material transfer in an environmentdependent manner," Stem Cell Reports, vol. 10, no. 2, pp. 406-421, 2018.

[163] S. D. Schwartz, J. P. Hubschman, G. Heilwell et al., "Embryonic stem cell trials for macular degeneration: a preliminary report," The Lancet, vol. 379, no. 9817, pp. 713-720, 2012.

[164] S. D. Schwartz, C. D. Regillo, B. L. Lam et al., "Human embryonic stem cell-derived retinal pigment epithelium in patients with age-related macular degeneration and Stargardt's macular dystrophy: follow-up of two open-label phase 1/2 studies," The Lancet, vol. 385, no. 9967, pp. 509-516, 2015.

[165] L. da Cruz, K. Fynes, O. Georgiadis et al., "Phase 1 clinical study of an embryonic stem cell-derived retinal pigment epithelium patch in age-related macular degeneration," Nature Biotechnology, vol. 36, no. 4, pp. 328-337, 2018.

[166] M. Mandai, A. Watanabe, Y. Kurimoto et al., "Autologous induced stem-cell-derived retinal cells for macular degeneration," The New England Journal of Medicine, vol. 376, no. 11, pp. 1038-1046, 2017.

[167] M. A. DeWitt, J. E. Corn, and D. Carroll, “Genome editing via delivery of Cas9 ribonucleoprotein,” Methods, vol. 121-122, pp. 9-15, 2017.

[168] X. H. Zhang, L. Y. Tee, X. G. Wang, Q. S. Huang, and S. H. Yang, "Off-target effects in CRISPR/Cas9-mediated genome engineering," Molecular Therapy Nucleic Acids, vol. 4, article e264, 2015.

[169] R. Peng, G. Lin, and J. Li, "Potential pitfalls of CRISPR/Cas9mediated genome editing," The FEBS Journal, vol. 283, no. 7, pp. 1218-1231, 2016.

[170] J. K. Yee, "Off-target effects of engineered nucleases," The FEBS Journal, vol. 283, no. 17, pp. 3239-3248, 2016.

[171] K. Nakajima, A. A. Kazuno, J. Kelsoe, M. Nakanishi, T. Takumi, and T. Kato, "Exome sequencing in the knockin mice generated using the CRISPR/Cas system," Scientific Reports, vol. 6, no. 1, article 34703, 2016.

[172] S. W. Cho, S. Kim, Y. Kim et al., "Analysis of off-target effects of CRISPR/Cas-derived RNA-guided endonucleases and nickases," Genome Research, vol. 24, no. 1, pp. 132-141, 2014.

[173] A. C. Komor, Y. B. Kim, M. S. Packer, J. A. Zuris, and D. R. Liu, "Programmable editing of a target base in genomic DNA without double-stranded DNA cleavage," Nature, vol. 533, no. 7603, pp. 420-424, 2016.

[174] N. M. Gaudelli, A. C. Komor, H. A. Rees et al., "Programmable base editing of $\mathrm{A} \bullet \mathrm{T}$ to $\mathrm{G} \bullet \mathrm{C}$ in genomic DNA without DNA cleavage," Nature, vol. 551, no. 7681, pp. 464-471, 2017. 


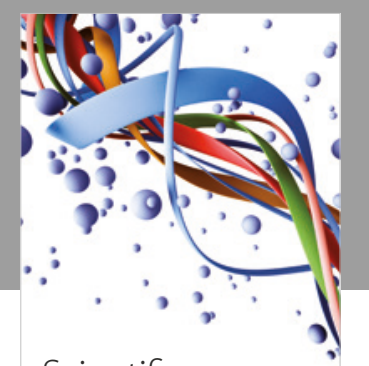

Scientifica
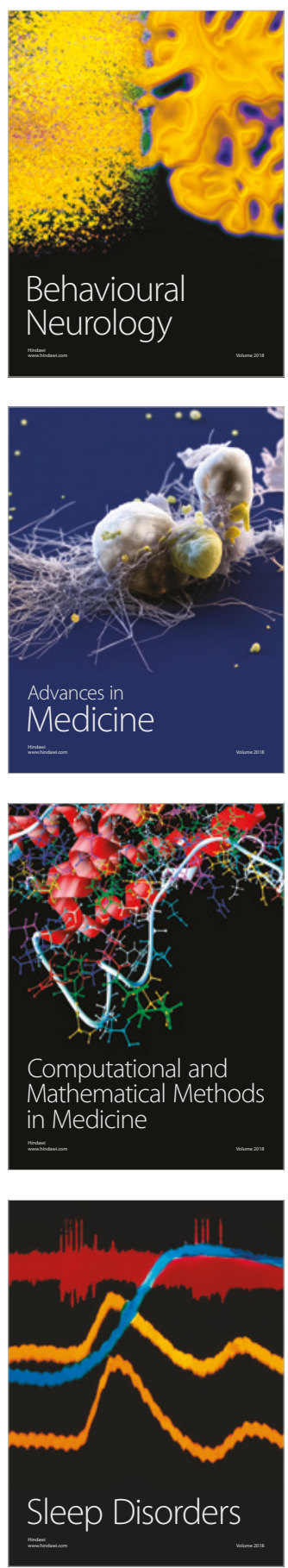

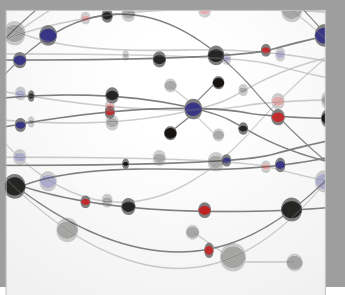

The Scientific World Journal

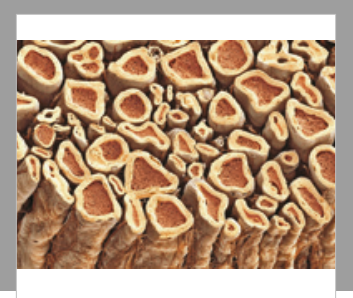

Case Reports in

Neurological Medicine

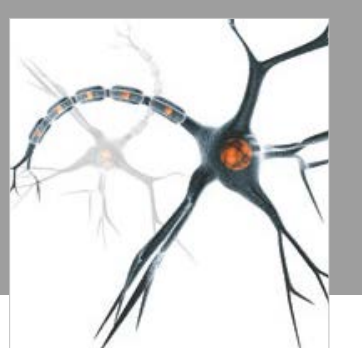

Neural Plasticity

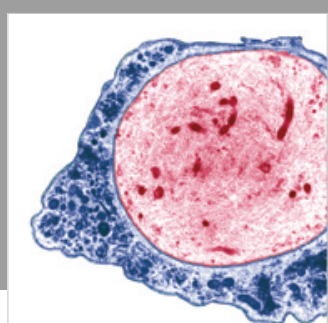

Multiple Sclerosis

International

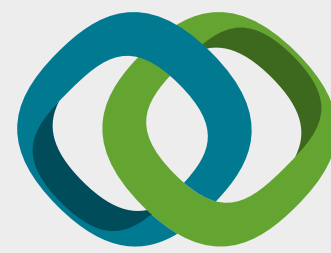

Hindawi

Submit your manuscripts at

www.hindawi.com
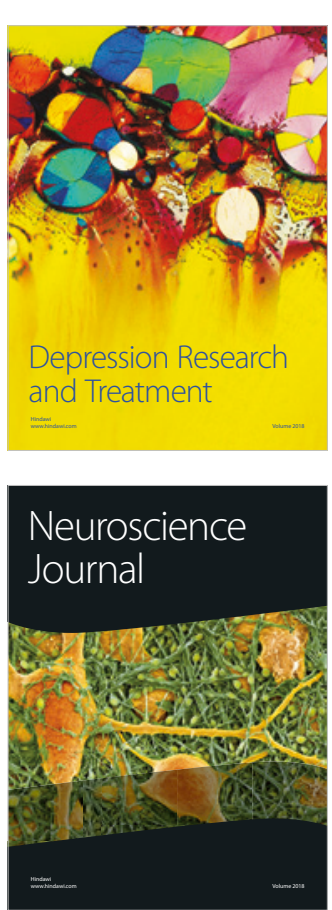

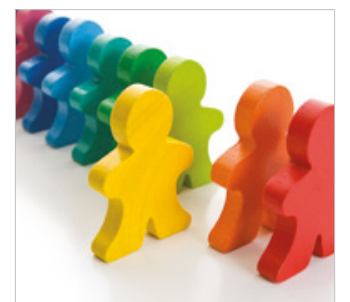

Autism

Research and Treatment
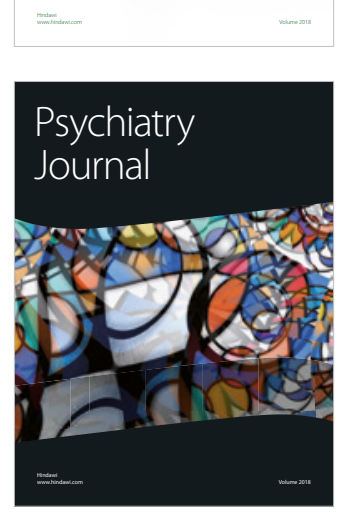
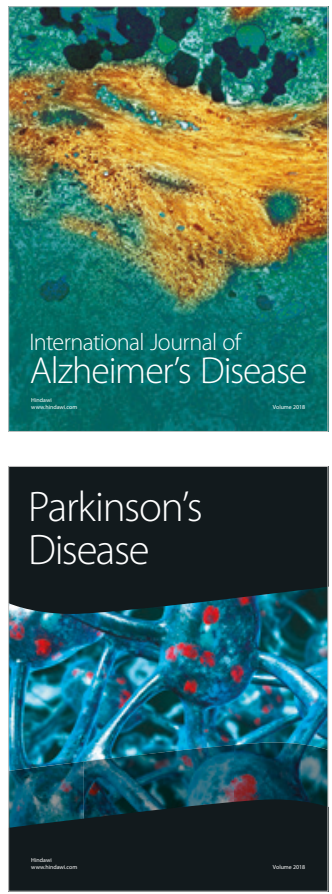
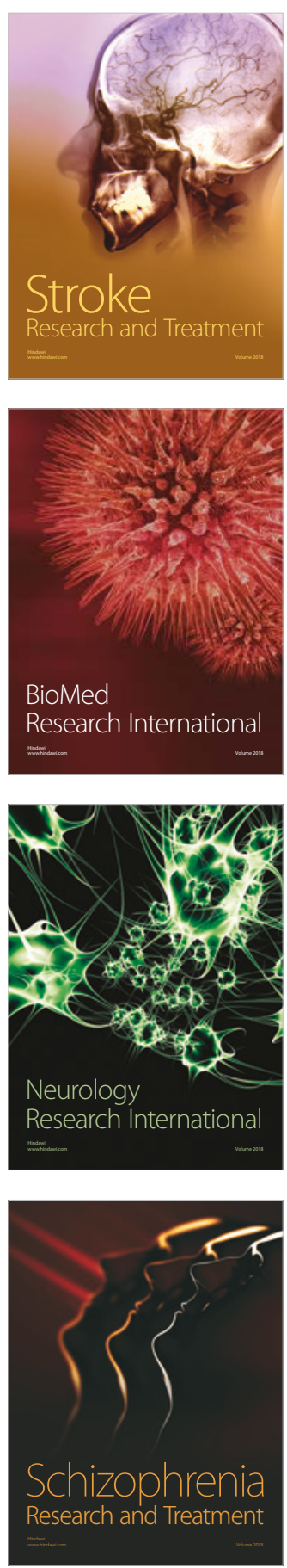